\title{
Robotic Heller-Dor myotomy: 10-year monocentric experience compared with POEM
}

Virginia Gallo ${ }^{*}$, Luca Morelli², Jacopo Viganò ${ }^{1}$, Luigi Pugliese ${ }^{1}$, Raffaele Fellegara ${ }^{1}$, Giuseppe Tartaglia ${ }^{1}$, Marika Sharmayne Milani ${ }^{1}$, Andrea Peri ${ }^{1}$

${ }^{1}$ Department of Surgery, Fondazione IRCCS Policlinico San Matteo, Pavia, Italy.

${ }^{2}$ Department of General Surgery, University of Pisa, Pisa, Italy.

\section{To Cite}

Gallo V, Morelli L, Viganò J, Pugliese L, Fellegara R, Tartaglia G, Milani MS, Peri A. Robotic Heller-Dor myotomy: 10-year monocentric experience compared with POEM. J Gastric Surg 2020; 2(3): 66-70

\section{Publication history}

Received: August 2, 2020

Accepted: August 6, 2020

Article in press: August 30, 2020

Published online: August 31, 2020

\section{*Correspondence to}

\section{Dr. Virginia Gallo}

Department of General Surgery

IRCCS Policlinico San Matteo

Viale Golgi 19

27100, Pavia, Italy

E-mail: virgygallo@yahoo.it

\section{ABSTRACT}

Achalasia is a rare motility disorder caused by an incomplete relaxation of the lower esophageal sphincter and loss of esophageal peristalsis. As a consequence, the bolus swallowing is hindered and the patients complain dysphagia, regurgitation, chest pain, respiratory symptoms and weight loss. Achalasia's treatment has been varied over time, from therapies aiming to relax the lower sphincter of the esophagus, including drugs and Botox injection or mechanical dilatations, to surgical myotomy. Robotic or laparoscopic Heller-Dor procedure is considered the gold standard surgical treatment for symptomatic achalasia as it is proved to be effective and safe.

As an alternative, Per-Oral Endoscopic Myotomy (POEM) was applied over the past decade, aiming to combine the same results of mini-invasive procedure to the advantages of endoscopic approach.

In this study, we are going to compare the medium-long term results of mini-invasive Heller-Dor procedure, routinely performed in our Department, with those of POEM reported in literature.

Keywords:

achalasia; robotic surgery; robotic Heller-Dor; POEM. 


\section{Background:}

Esophageal achalasia is a primary motility disorder characterized by an incomplete relaxation of the lower esophageal sphincter and the absence of peristalsis. Therefore, patients complain with obstructive symptoms like dysphagia, regurgitation, chest pain and weight loss. Respiratory manifestations such as nocturnal cough, dyspnea and pneumonia may occur in advanced stages. Achalasia is a chronic disease that leads to loss of functional properties and anatomical alterations of the esophagus; usually it is not diagnosed in early phases, but it is important to recognize it before some irreversible changes occur [1].

Obstructive symptoms are suggestive for the diagnosis, which can be confirmed by instrumental exams such as esophageal manometry, fluoroscopy with contrast swallow and endoscopy. According to 2019 Seoul Consensus on Esophageal Achalasia Guidelines, manometry is considered essential for the diagnosis and neither fluoroscopy nor EGD are sensitive enough to confirm the disease [1].

Currently, a specific therapy targeting the causative mechanism of achalasia is not available, since its pathophysiology is unknown; hence, treatment of achalasia aims to palliate dysphagia and possible associated complications by reducing esophagogastric junction (EGJ) pressure, in order to allow the passive transit of the bolus into the stomach [1-3].

Oral drugs reducing LES pressure are administered to patients unsuitable for endoscopic or surgical procedures and their effect is temporary, from 90 minutes of nitrates to 120 minutes of calcium channel blockers. Also, botulinum toxin injection shows short-term benefits; however, in patients with poor general conditions, repeated administration should be considered [1].

Pneumatic balloon dilatation (PD) is a valuable alternative to surgical treatment of achalasia, as it can provide good long-term results according to retrospective analysis [1]. However up to $30 \%$ of patients experience gastroesophageal reflux after dilatation, which may cause recurrent LES stenosis and dysphagia [4]. Nevertheless, many studies report that PD is as effective as surgical treatment, i.e. Heller-Dor myotomy, even after 2 years of follow up, while some authors find outcome differences according to achalasia severity, being surgical intervention more effective in symptoms remission rate than dilatation for more severe disease manifestations [1].

Heller-Dor myotomy requires full-thickness section of the muscular layers of the esophagus, extended $5 \mathrm{~cm}$ above the EGJ and $2 \mathrm{~cm}$ onto the stomach. Usually, the myotomy is followed by Dor fundoplication to prevent gastro-esophageal reflux onset [5].

Heller-Dor procedure performed in mini-invasive fashion is nowadays considered the gold standard approach for primary treatment of fit-for-surgery patients affected by achalasia or for patients not responsive to pharmacological or endoscopic therapies $[5,6]$.

With technological improvement, robotic HellerDor (RAHD) has gained more interest thanks to the implementation of stereoscopic tridimensional optical system and dexterity, increasing surgeon's sensibility in performing the myotomy and reducing possible complications such as gastroesophageal perforation compared to laparoscopic approach. In this regard, Kim et al. [7] confirmed a less frequent iatrogenic gastroesophageal perforation with robotic approach and observed a reduced incidence of recurrent achalasia, due to the agility conferred by wrist-like movements of robotic tools, which allow to better peel muscle fibers laterally preventing them from healing together.

Recent advantages in endoscopic procedures, which are even less invasive and painful than surgical interventions performed laparoscopically, have introduced a novel technique of Per-Oral Endoscopic Miotomy (POEM) among the therapeutic options for achalasia. It is an advanced endoscopic procedure which consists in creating a submucosal tunnel from the middle third of the esophagus to the proximal stomach, followed by a myotomy extended from $2 \mathrm{~cm}$ below initial mucosal incision towards the cardia [8,9]. At the beginning POEM was criticized for the absence of any anti-reflux valve system confection, which may result in post-procedural gastro-esophageal reflux occurrence. Recently, POEM results in terms of success rate, adverse effects and postprocedural reflux onset have been evaluated by many systematic reviews and metanalyses, showing the noninferiority of this technique to surgical Heller-Dor [1013].

Hence, we present our 10-year experience in the treatment of achalasia with RAHD, submitting our surgical and functional results aiming to compare them to those of POEM reported in literature.

\section{Methods:}

Patients treated with robot-assisted Heller-Dor myotomy (RAHD) in our Department of General Surgery, IRCCS Policlinico San Matteo, Pavia, from January 2010 to January 2020 were retrospectively extracted from informatic database. All patients were diagnosed with esophageal achalasia based on clinical presentation and radiological or manometrical preoperative exams.

Data regarding preoperative clinical characteristics, intraoperative and post-operative information were collected, therefore including age, sex, BMI, ASA score, operative time, previous surgical interventions and complained symptoms.

With the aim to objectify the severity of dysphagia and others achalasia related symptoms Eckardt score (ES) was used, which assigns a score from 0 to 3 on the basis of patient's self-reported response to four main disease manifestations (dysphagia, regurgitation, chest pain and weight loss), resulting in a total score ranging from 0 to 12 . Follow up was accomplished via telephone on April 2020 administering ES again along with Gastroesophageal Reflux Disease Questionnaire (GERD-Q) to evaluate potential achalasia recurrence or reflux disease late onset [14]. GERD-Q assesses the probability of having gastro-esophageal reflux (RE) considering symptoms like heartburn, regurgitation, epigastric pain, nausea, sleep disorders and use of PPI and rating them from 0 to 3 score. The final score ranges from 0 to 18; many studies suggest that the higher is the score, the greater is the possibility of RE. Moreover, further studies demonstrate that the risk of reflux is low 
when GERD-Q is smaller than 8 [15].

Results obtained from statistical analysis of our data were matched with those obtained after RAHD and POEM reported in literature so far.

\section{Results}

During the study period, a total of 55 patients were treated with RAHD myotomy, 27 males and 28 females, with a mean age of $57,1 \pm 19,1$ years.

The mean ASA score was $1,85 \pm 0,67$, being ASA 2 class mostly represented ( 29 patients, $52,2 \%$ ); mean body mass index (BMI) was $23,4 \pm 9,66 \mathrm{Kg} / \mathrm{m} 2$. Mean operative time was $165 \pm 40,5 \mathrm{~min}$. Neither intraoperative nor postoperative complications were recorded, except for one episode of recurrent vomiting following diet reintroduction in a patient with megaesophagus affected by esophageal candidiasis, successfully managed conservatively. Mean hospital stay after surgery was $4,5 \pm 3$ days; most patients were discharged in third POD $(20,36,3 \%)$. Only the aforementioned patient was discharged after 25 days after surgery.

Mean Eckardt score administered preoperatively to 53 on 55 patients was $8,2 \pm 1,7$ (range 3-12).

Follow-up lasted from 3 to 87 months, with an average of $36,7 \pm 26,2$ months. 2 patients died and 3 were lost to follow-up. Therefore, post-operative questionnaires were administered to 50 on 55 patients. Mean postoperative Eckardt score was $1 \pm 1,53$, resulting in a good symptoms resolution after surgical procedure during the follow up period examined. The difference between pre and post-operative Eckardt score is 7 that is statistically significative $(p<0,001)$.

Mean GERD-Q score was $6 \pm 0.91$, ranging from a minimum of 5 and a maximum of 10: in particular, 29 $(58 \%)$ patients were assigned a score of $6,11(22 \%)$ a score of $7,5(10 \%)$ a score of $5,4(8 \%)$ a score of 8 and 1 $(2 \%)$ a score of 10 . Considering 8 points as a cut off for RE diagnosis, we concluded that 5 patients $(10 \%)$ of our case study treated with robotic Heller-Dor procedure suffered from post-operative gastro-esophageal reflux.

\section{Discussion}

Robotic Heller-Dor myotomy is a safe and feasible procedure for the treatment of achalasia. Technological innovations introduced by robotic surgery such as tridimensional magnified image and articulated instruments allowed a reduction of intraoperative complications (i.e. perforations) and better results in terms of symptoms relief than laparoscopic approach. A monocentric study by Pallabazzer et al. [16] showed no intraoperative complication in a series of 66 patients undergone to RAHD; the same results were reported by Galvani et al [17]. Indicative is a multicentric study by Melvin and colleagues [18] who submitted a series of 104 robotic Heller-Dor without a single case of perforations. Kim et al. [7] described only one esophageal perforation on a series of 37 patients treated with robotic approach and 4 perforations in the laparoscopic group; three of these patients required conversion to open surgery. Our study reflects the results reported in literature as we described no intraoperative complications and no need to laparotomic conversion. Moreover, we did not face any postoperative complications requiring surgical reintervention, consistent with other studies' results. All patients of our series interviewed at follow up declare dysphagia resolution, which is reflected also by the significative reduction of the Eckardt score $(\mathrm{p}<$ $0,001)$. This ES decrease is observable also in 26 patients with > 24 months follow-up, whose mean ES was 1, suggesting a long-term results maintenance. Perry et al. [19], who described dysphagia relief even after 9 years of follow up in $84 \%$ of patients, support long-term efficacy of RAHD.

Up to now, probably due to the rarity of the disease, there are only few studies with small cohort of patients comparing outcomes of LHM vs POEM; even more so, studies comparing benefits and results of RAHD vs POEM are less. A recent retrospective study evaluating the intra-operative advantages, short-term and long-term results of robotic vs laparoscopic Heller Dor, showed a lower incidence of intraoperative complications, such as perforation and a lower rate of achalasia recurrence with robotic technique [19]. According to this, it would be expected that RADH would show better results at least in terms of intraoperative adverse events than LHM when compared to POEM.

A multicenter, randomized controlled trial comparing 112 POEM and 109 LHM plus Dor fundoplication stated a comparable success rate at 2 years follow up but a higher incidence of intraoperative complications in LHM group (7,3\% vs 2,7\%) [20]. This is true when POEM is performed by experienced endoscopists: in fact, Ren et al. [21] showed a higher complication rate in POEM, probably due to learning curve. On the other hand, more trained endoscopists like Inoue et al. [22] and Zhang et al. [23] showed a very low complication rate $(3,2 \%$ and $3,3 \%$ respectively).

However, a review by Crespin and colleagues [6] reported a variable rate of complications with POEM from 0 to 30\%; according to a 2018 review by Parsa et al. [24], the incidence of adverse effects after POEM varies from 0 and $72,2 \%$. This great variability can be explained by the heterogeneity of studies reporting adverse effects and different definitions of complications used. Major complications are emphysema, pneumoperitoneum, mediastinal emphysema, and pneumothorax that are generally recognized and managed during the procedure without sequelae. Again, a comprehensive multicenter study of 1826 patients undergone POEM reports a complication rate of $7,5 \%, 0,5 \%$ of which of severe entity and concludes that POEM is a safe procedure when performed by expert operators in tertiary centers [25].

The efficacy of POEM in relieving dysphagia and other achalasia-related symptoms is well known by now. Previous metanalyses by Akintoye and colleagues [26] reported a clinical success rate of $98 \%$ at 12 months of follow up, defined by an ES reduction from 6.9 to 1 . In 2019 Consensus on esophageal achalasia guidelines the efficacy rate was 92,8\% [1]; other meta-analyses concluded that POEM has the same efficacy of LHM, even in long-term follow up $87 \%$ after a median follow up of 49 months) [27]. The incidence of postoperative RE following POEM is a matter of debate. Many studies aiming to evaluate this complication report variable results depending on the modality of detecting RE (clinic, Ph-testing, endoscopy); a recent meta-analysis 
reports a post-POEM reflux rate from 7,5 to $47 \%$ whereas in surgical myotomy reflux rate ranges from $5,6 \%$ to $43 \%$, statistically not different [28]. In our series, only 5 patients $(10 \%)$ complained with symptoms ascribable to esophageal reflux reflected by a GERD-Q score $\geq 8$. However, a dissociation between symptoms of gastroesophageal reflux and endoscopic esophagitis findings is present: one of the meta-analyses with the largest number of patients concluded that both POEM and surgical myotomy plus Dor fundoplication cause comparable rates of post-procedural reflux-related symptoms, but there was a greater number of patients with esophagitis endoscopically detected in POEM group $(22,4 \%$ vs $11,5 \%)$ [29].

Recently, POEM has been considered as a rescue endoscopic therapy for patients who failed surgical Heller-Dor, reporting high success rate in terms of dysphagia's resolution in many studies [30,31]. In fact, redo Heller-Dor is a challenging procedure due to fibrosis and adhesions from the previous surgery which may lead to intraoperative complications such as gastroesophageal perforation; endoscopic reintervention allows to overcome these issues, reducing operative time and blood loss and to access to the entire esophagus in order to perform longer myotomy if needed [32].

\section{Conclusions}

POEM proved to be an effective technique in relieving major symptoms of achalasia with a success rate comparable to Heller-Dor procedure.

However, we believe that some considerations need to be addressed.

Incision-free nature of POEM is believed to be the main advantage over laparoscopic or robotic approach, since it can overcome post-operative pain of surgical procedures. In our experience, patients after RAHD usually complain with weak and short-lasting pain, perfectly manageable with analgesics and they can get up from bed by the same afternoon/evening. Besides, patients are safely given liquid diet in POD 1 and solid diet in POD 2 and they are discharged on POD 3.

Post-procedural care after POEM is different according to clinicians' attitude: some practitioners perform gastrografin swallow study to check integrity of mucosal suture and give patients soft diet for at least two weeks before starting a regular diet; others discharge patients on POD 1, even if some patients feel well enough to leave in the evening; they reserve $\mathrm{X}$-ray with gastrografin swallow to patients complaining with more pain than expected, in case of dysphagia persistence or challenging mucosal suturing [8].

It is evident that post-operative management after RAHD is more standardized in Italy and worldwide, as a consequence not only of the longer experience with this approach but also of the low probability to incur late complications such as bleeding or perforation which, on the other hand, may occur with clips abruption after POEM.

Moreover, is well recognized that POEM is a complex procedure reserved to experienced endoscopists and that should be performed in high volume centers only in order to guarantee the best outcome for patients. Endoscopists must be an expert in esophageal pathology and must be familiar with main operative procedures such as submucosal dissection as well as being able to face intraprocedural complications $[9,21,24,33]$.

Training process should start from watching the procedure performed by more experienced operators familiarizing with required equipment. Thereafter, endoscopists should perform POEM on animal models to develop the necessary skills until they can access to tertiary centers [34].

In Italy there are few referral centers focused on POEM as achalasia in mainly treated surgically; the Da Vinci Si robotic system is widely spread on national area and its use is mainly intuitive without long and demanding training. The availability of double console allows training of naïve surgeons, enabling them to perform some easy surgical steps of Heller-Dor procedure that lacks of particular critical issues after all.

Most studies mentioned before report comparable esophageal reflux rate after POEM and Heller Dor; however, these results are conditioned by different methods to test the actual reflux presence and therefore objective studies are strongly recommended [24]. Anyway, the section of the lower esophageal sphincter predisposes to reflux especially if it isn't associated to anti-reflux valve; in this way, it is reasonable to think that almost the totality of patients undergoing POEM suffers of RE, regardless of whether it is symptomatic or not, with high probability to develop reflux associated complications.

For these reasons, we do not consider POEM as a convenient alternative technique to surgical Heller-Dor as it does not bring remarkable advantages in terms of effectiveness, intra e post-procedural complications, reflux rate, operative time and technical ease compared to RAHD. However, we agree with POEM as a rescue procedure after Heller-Dor's failure, as it allows the operator to revise the site of myotomy without encounter the difficulties related to previous surgery.

\section{Acknowledgements}

None

\section{Contributors}

VG wrote the manuscript and analyzed patients' data. JV, RF and GT had a fundamental contribution in drafting the database. LP, LM and MSM reviewed the manuscript.

\section{Funding}

No funding was received for this study.

\section{Competing interests}

The authors declare that they have no competing interests.

\section{Availability of data and materials}

The datasets used and/or analyzed during the current study are available from the corresponding author on reasonable request.

\section{Ethics approval}

Not applicable 


\section{Provenance and peer review}

Not commissioned; externally peer reviewed.

\section{Open access}

This is an Open Access article distributed in accordance with the Creative Commons Attribution NonCommercial (CC BY-NC 4.0) license, which permits others to distribute, remix, adapt, build upon this work noncommercially, and license their derivative works on different terms, provided

the original work is properly cited and the use is non-commercial. See: http://creativecommons.org/ licenses/by-nc/4.0/

\section{References}

[1] Hye-Kyung Jung, Su Jin Hong, Oh Young Lee, John Pandolfino, Hyojin Park, Hiroto Miwa, Uday C Ghoshal, Sanjiv Mahadeva, Tadayuki Oshima, Minhu Chen, Andrew S B Chua, Yu Kyung Cho, Tae Hee Lee, Yang Won Min, Chan Hyuk Park, Joong Goo Kwon, Moo In Park, Kyoungwon Jung, Jong Kyu Park, Kee Wook Jung, Hyun Chul Lim, Da Hyun Jung, Do Hoon Kim, Chul-Hyun Lim, Hee Seok Moon, Jung Ho Park, Suck Chei Choi, Hidekazu Suzuki, Tanisa Patcharatrakul, Justin C Y Wu, Kwang Jae Lee, Shinwa Tanaka, Kewin T H Siah, Kyung Sik Park, Sung Eun Kim, and The Korean Society of Neurogastroenterology and Motility. 2019 Seoul Consensus on Esophageal Achalasia Guidelines J Neurogastroenterol Motil. 2020 Apr 30; 26(2): 180-203.

[2] Barbieri L.A., Hassan C., Rosati R., Fumagalli Romario U., Correale L., Repici A. Systematic review and meta-analysis: Efficacy and safety of POEM for achalasia. United European Gastroenterology Journal 2015 3(4):325-334

[3] Teitelbaum E.N., Soper N.J., Arafat F.O., Santos B.F., Kahrilas P.J., Pandolfino J.E., Hungness E.S. Analysis of a learning curve and predictors of intraoperative difficulty for Peroral Esophageal Myotomy (POEM). J Gastrointest Surg (2014) 18:92-99

[4] Boeckxstaens G.E., Annese V., Varannes S.B. et al. Pneumatic Dilation versus Laparoscopic Heller's Myotomy for Idiopathic Achalasia. N Engl J Med. 2011;364(19):1807-1816.

[5] Cheguevara A., Finnerty B., Abelson J.S., Zarnegar R. Robotic-assisted Heller myotomy: a modern technique and review of outcomes. J Robot Surg 2015 Jun;9(2):101-8.

[6] Crespin O.M., Liu L.W.C., Parmar A., Jackson T.D., Hamid J., Shlomovitz E., Okrainec A. Safety and efficacy of POEM for treatment of achalasia: a systematic review of the literature. Surg Endosc (2017) 31:2187-2201

[7] Kim S.S., Guillen-Rodriguez J., Little A.J. Optimal surgical intervention for achalasia: laparoscopic or robotic approach. Journal of Robotic Surgery (2019) vol 13, pages 397-400

[8] Holmstrom A.L., Hungness E.S. POEM for Esophageal Achalasia. Journal of laparoendoscopic \& advanced surgical techniques (2020) Volume 00, Number 00

[9] Banks M., Sweis R. POEM and the management of achalasia. Frontline Gastroenterology 2017; 8:143-147

[10] Werner Y.B., Costamagna G., Swanström L.L. et al. Clinical response to peroral endoscopic myotomy in patients with idiopathic achalasia at a minimum follow-up of 2 years. Gut 2016; 65:899-906

[11] Hungness E.S., Sternbach J.M., Teitelbaum E.N. et al. Peroral Endoscopic Myotomy (POEM) after the learning curve: durable long-term results with a low complication rate. Ann Surg 2016; 264:508-17.

[12] Bhayani N.H., Kurian A.A., Dunst C.M. et al. A comparative study on comprehensive, objective outcomes of laparoscopic Heller myotomy with per-oral endoscopic myotomy (POEM) for achalasia. Ann Surg 2014; 259:1098-103

[13] Ujiki M.B., Yetasook A.K., Zapf M. et al. Peroral endoscopic myotomy: a short-term comparison with the standard laparoscopic approach. Surgery 2013; 154:893-7; discussion 897

[14] Jonasson C., Wernersson B., Hoff D.A.L., Hatlebakk J.G. Validation of the GerdQ questionnaire for the diagnosis of gastrooesophageal reflux disease. Aliment Pharmacol Ther. 2013;37(5):564572

[15] Zavala-Gonzales M.A., Azamar-Jacome A.A., MeixueiroDaza A. et al. Validation and diagnostic usefulness of gastroesophageal reflux disease questionnaire in a primary care level in Mexico. J Neurogastroenterol Motil. 2014;20(4):475-482.

[16] Pallabazzer G., Peluso C., de Bortoli N., Solito B. D’Imporzano S., Belluomini M.A., Bellomini M.G., Giusti P., Gianetri D., Santi S. Clinical and pathophysiological outcomes of the robotic assisted Heller-Dor myotomy for achalasia: a single center experience. Journal of Robotic Surgery (2020); 14:331-335

[17] Galvani C., Gorodner M.V., Moser F., Baptista M., Donahue P., Horgan S. Laparoscopic Heller myotomy for achalasia facilitated by robotic assistance. Surg Endosc (2006); 20:1105-1112

[18] Melvin W.S., Dundon J.M., Talamini M., Horgan S. Computer-enhanced robotic telesurgery minimizes esophageal perforation during Heller myotomy. Surgery (2005); 138:553-558

[19] Perry K.A., Kanji A., Drosdeck J.M., Linn J.G., Chan A., Muscarella P., Melvin W.S. Efficacy and durability of robotic Heller myotomy for achalasia: patient symptoms and satisfaction at longterm follow-up. Surg Endosc (2014); 28:3162-3167

[20] Werner Y.B., Hakanson B., Martinek J., Repici A., Burkhard H.A. von Rahden, Albert J. Bredenoord A.J., Bisschops R., Messmann H., Vollberg M.C., Noder T., Kersten J.F., Mann O. et al. Endoscopic or Surgical Myotomy in Patients with Idiopathic Achalasia N Engl J Med 2019; 381:2219-2229

[21] Ren Z., Zhong Y., Zhou P., Xu M., Cai M., Li L., Shi Q., Yao L. Perioperative management and treatment for complications during and after peroral endoscopic myotomy (POEM) for esophageal achalasia (EA) (data from 119 cases). Surg Endosc 2012 Nov;26(11):3267-72

[22] Inoue H., Sato H., Ikeda H., Onimaru M., Sato C., Minami H. et al. Per-Oral Endoscopic Myotomy: A Series of 500 Patients. J Am Coll Surg. 2015; 221:256-64

[23] Zhang X.C., Li Q.L., Xu M.D., Chen S.Y., Zhong Y.S., Zhang Y.Q. et al. Major perioperative adverse events of peroral endoscopic myotomy: a systematic 5-year analysis. Endoscopy. 2016; 48:967-78

[24] Parsa N., Khashab M.A. POEM in the Treatment of Esophageal Disorders. Curr Treat Options Gastro (2018) 16:27-40

[25] Haito-Chavez Y., Inoue H., Beard K.W., Draganov P.V,. Ujiki M., Rahden B.H.A. et al. Comprehensive analysis of adverse events associated with per oral endoscopic myotomy in 1826 patients: an international multicenter study. Am J Gastroenterol. 2017;112(8):126776

[26] Akintoye E., Kumar N., Obaitan I., Alayo Q.A., Thompson C.C. Peroral endoscopic myotomy: a meta-analysis. Endoscopy 2016; 48:1059-1068

[27] de Pascale S., Repici A., Puccetti F., Carlani E., Rosati R., Fumagalli U. Peroral endoscopic myotomy versus surgical myotomy for primary acha-lasia: single-center, retrospective analysis of 74 patients. Dis Esophagus 2017; 30:1-7

[28] Martins R.K., Ribeiro I.B., Hourneaux De Moura D.T., Hathorn K.E., Bernardo W., Hourneaux De Moura E.G. Peroral (POEM) or surgical myotomy for the treatment of achalasia: a systematic review and meta-analysis. Arq Gastroenterol Jan-Mar 2020;57(1):79-86

[29] Schlottmann F., Luckett D.J., Fine J., Shaheen N.J., Patti M.G. Laparoscopic Heller Myotomy Versus Peroral Endoscopic Myotomy (POEM) for Achalasia: A Systematic Review and Meta-analysis. Ann Surg. 2018; 267:451-60

[30] Vigneswaran Y., Yetasook A.K., Zhao J.C. et al. Peroral endoscopic myotomy (POEM): feasible as reoperation following Heller myotomy. J Gastrointest Surg 2014; 18:1071-1076.

[31] Zhou P.H., Li Q.L., Yao L.Q. et al. Peroral endoscopic remyotomy for failed Heller myotomy: a prospective single-center study. Endoscopy 2013;45:161-166

[32] Ngamruengphong S., Inoue H., Ujiki M.B., Patel L.Y. et al. Efficacy and Safety of Peroral Endoscopic Myotomy for Treatment of Achalasia After Failed Heller Myotomy. Clinical Gastroenterology and Hepatology 2017; 15:1531-1537

[33] Marano L., Pallabazzer G., Solito B., Santi S., Pigazzi A., De Luca R., Biondo F.G., Spaziani A., Longaroni M., Di Martino N., Boccardi V., Patriti A. Surgery or Peroral Esophageal Myotomy for Achalasia A Systematic Review and Meta-Analysis. Medicine (Baltimore) 2016 Mar;95(10):e3001.

[34] Ahmed Y., Othman M.O. Peroral endoscopic myotomy (POEM) for achalasia. J Thorac Dis 2019 Aug;11(Suppl 12):S1618-S1628. 